\title{
Reducing Sensors according to a VectorsAnalysis of stored measurements (ReSeVA)
}

\author{
Ammar Suhail Balouch \\ Associate Prof. Dr. Eng., Database Research Group \\ Department of Computer Science \\ University of Rostock
}

\begin{abstract}
The recognition of motion is widely used in games development field but it is active too in care systems. Recognition of motion according measurements needs data (values) from many sensors, like Position, Velocity, Acceleration, Orintation, etc. We have two major ways to determine which placements of sensors on the body are required to recognize the motion. The first way connects its work with the results of other science branches like sports science and game development. The other one depends on the following strategy. Many sensors were placed on the body, without the knowledge, which sensors are required. Then according an analysis of the stored data for each sensors, the behavioral similarity of these sensors will be extracted.The target of both ways is to reduce the cost of building a suit of sensors, and simultaneously to keep the results of the recognition of motion correct.In this paper we follow the second way and define a new regression analysis "ReSeVA" depending on vector definition(on its angles and longs) and on the principle of Newton's law of metion.
\end{abstract}

Keywords: recognition of motion, Data analysis, Reduction of sensors, linear Regression, Vector, Distance matrix analysis.

\section{INTRODUCTION}

The care systems are today from the most important things in medicine and computer science fields to give the strongly sick people some safety and to eliminate the dangerous of their lives. For this reason many researchers have developed systems to recognize the motion of people and according this recognition can the system send warning signals if needed. The recognition of motion is widely used in games development field and in other scientific fields. Recognition of motion needs values (measurements) from many sensors, like Position, Velocity, Acceleration, Orintation, etc.

There are two major ways to determine which placements of sensors on the body are required to recognize the motion. The first way connect its work with the results of other science branches like sports science and game development. The other one depends on the following strategy. Many sensors were placed on the body, without the knowledge, which sensors are required. Then according an analysis of the stored data for each sensors, the behavioral similarity of these sensors will be extracted. The target of both ways is to reduce the cost of building a suit of sensors, and simultaneously to keep the results of the recognition of motion correct.

Our methodology is located at the second way. With this methodology we define a model to extract the similarity of sensors measurements without any knowledge of other scientifical field. This model depends on the angle and on the long of all vector of each sensors.

This article is organized as follows: In section 2 we list related works with a short summary. The development fields toreduce the number of sensors is to find in section 3.The new analysis trend is presented in section 4. We summarize the most importantpoints of the article in last section 5. 


\section{RELATED WORK}

To our knowledge there are no papers that have presented mothods or techniques that reduce the number of sensors according to an analysis of raw measured data with using a linear regression. Therefore we will give some papers, which have described technique depending on these both direction seperatly, the reducing the number of sensors and the linear regression analysis.

The studies to reduce the number of sensors is more common in industrial fields and in game developments. Englert et al. present in [1] a system named SensiMateto estimate a usere's energy footprint. With this system they relied on sound samples from the user's envirnoment that have been recorded using mobile device. SensiMate consist of five components arranged in a processing pipeline. In the first stage of this pipeline the required sensor data will be captured and a raw data streamoutput.In the field of mobile air quality sensor network applications the authors in [2] exploited the correlation between differenet types of sensors coused by cross sensitivity to helo identify and correct the outlier readings. In this work a Bayesian belief network based system has been designed to detect and recover the abnormal readings, methods have been developed to update the sensor calibration functions in-field without requirement of ground truth and a real-world mobile sensor network has been deployed using the 15 custom-built M-Pods to verify their assumptions and technique.Kim et al. present in [3] an animation system that reproduces full-body character animation. This system receive the measurments from five 3D motion sensors on the performer. In this work is the Canonical-Correlation-Anaylsis-based regression employed as the model that effectively covers a wide range of motion.Badler et al. have presented in their paper [4] realistically recreate human postures while minimally encumbering the operator. In this work the authors have fixed a number of 6 DOF sensors to capture full body standing postures and they meant that this number is minimal to illustrate the motion of a human body. Altun and Barshan have provided in [5] a comparative study on the different techniques of classifying human activitiesusing accelerometers, gyroscopes, and magnetometers. This study depends on five sensors modules, each of which includes a triaxial accelerometer, a triaxial gyroscope, and a triaxial magnetometer. In [6] have Jones and Shao developed temporal segementation system that recognizes the human action and based on person tracking and a novel segmentation algorithm. The optical flow PCA and linear regression error estimation were applied in this method to get a metric, which can be used to split an action into several more easily recognized subactions. They mean thatsplitting human tracks into linear motions may also reduce the complexity of localisation methods. There are many works and papers [7-12] that present a systems to recognize a motion of human body but all these papers do not try to reduce the number of sensors but they fixed a number of sensors or from other devices like a camera by using regression analysis or other techniques. In the game industry field there are a big number of publications that provide technologies to recognize and analyse a human motion. In [13] the authors have provided a motion sensging's boon in the gaming insudtry depending on a technology that assessment of the future of motion sensing technology. Premaratne presented in [14] the historical development of hand gesture recognition. While Hwanlk Jo et.al. present in [15] a method to compensate for the location of the user and motion data that can't be recognized by one kinect using acquired by multi kinects and implemented the human body trace system for multiple users.In [16] present Liguang Xie et.al. estimation framework of motion that utilizes a small set of low-cost by using 3D acceleration sensors. Scholz et.al. describe in [17] an approach for generation of locomotion for an autonomous humanoid robot with condsideration on the minimal sensor input.

\section{DEVLOPMENT FIELDS}

Our first thought to reduce the amount of sensors according their measurements was depending on the distance matrix analysis with its represented graphs (One graph for each measuring time for each sensors). According the comparison of these graphs will the behavioral similarity be determinated. 


\section{Distance Matrix Analysis}

Let us look at the position's values of each sensros (Figure 1). We consider only these values

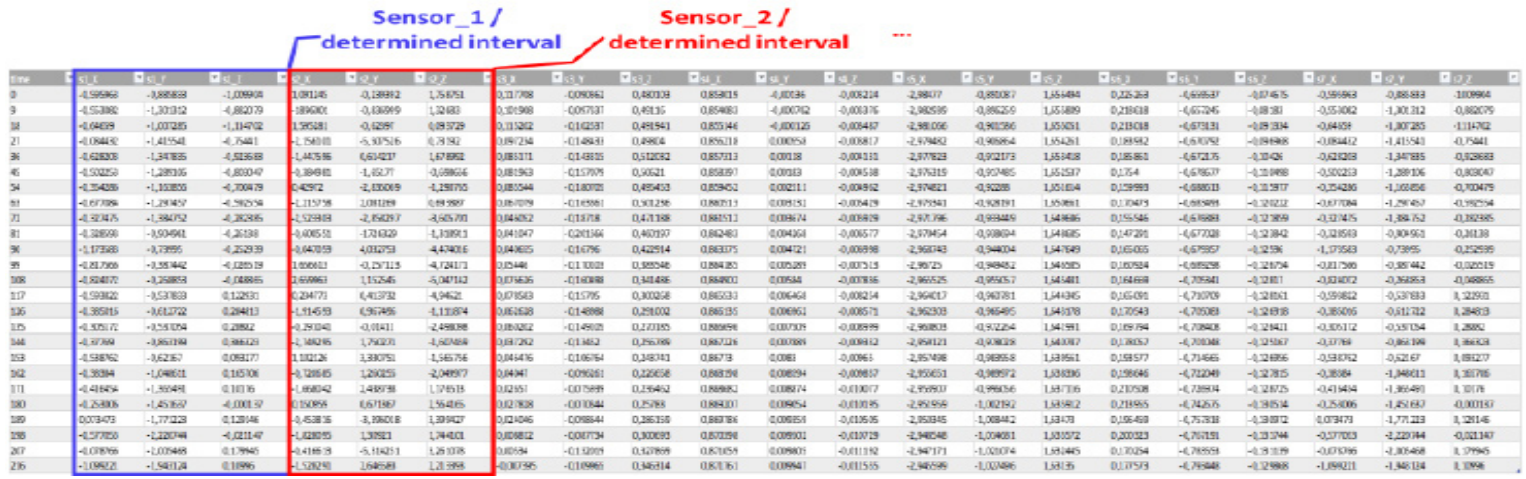

Fig1. Position values table of sensors

to compute the distance matrixs between all sensors in each measuring time and to represent their graphs (Figure 2).
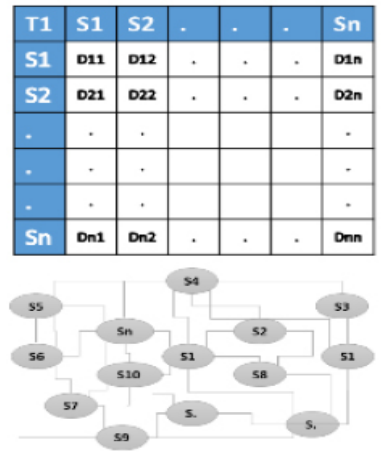
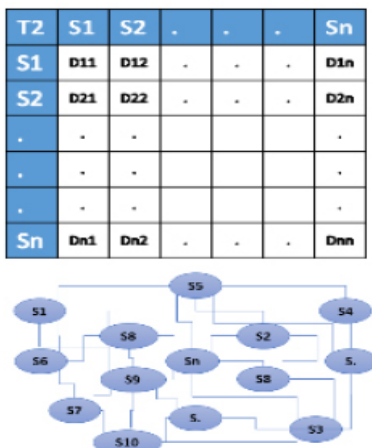

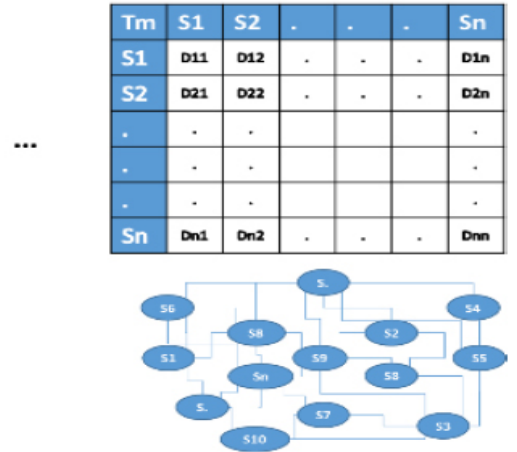

Fig2. Abstract of Distance Matrix Analysis

This methodology is very expensive and need a graph comparison technique to result its descision.From the idea of distance matrix anaylsis and due to its complexity, we came up with an idea, which is dependet on the definition of motion by newton.

\section{Motion Definition by Newton}

The motion equation is a linear circular equation determining by Angels (Figure 3):

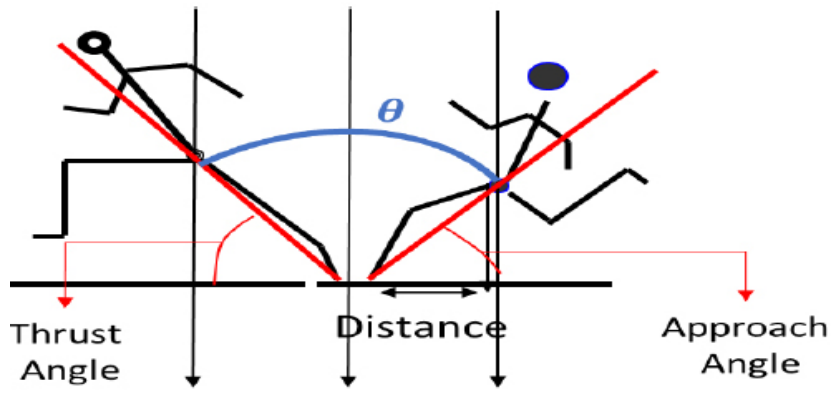

Fig3. Motion determining by angels 
The motion can be determined by compute the approach angle and thrust angle. For this principle we added the mechanism that we define vectors between a coordinates-center and the center of the body for the different positions and consider on the angle $(\theta)$ between two vectors and later to complete the model we compute the angles $(\alpha, \beta, \gamma)$ between vectors and corrdinates-axes $(O X, O Y, O Z)$ respectively and calculate the long of each vector, because the angle and the long of vectors together can recognize the motion not only in walk but in other motion situations (Sit, Walk-Sit, stand, ... etc.).

\section{Motion Vector Analysis (ReSEVA)}

The first step in our analysis is to derive the vector values from the positions sotred values. We covert the stored sensors values table in new table, which consist of only two columns. One contains the angel-values between two successively vectors and the second storages the long of the accordingly vector.

\subsection{Converting sensor'values}

A vector will be defined for each sensors in each measuring time $\overrightarrow{U_{s_{j}}}$, where $\boldsymbol{j}$ is for the sensors and $\boldsymbol{i}$ for the measuring times (Figure 4).

\begin{tabular}{|c|c|c|c|c|c|c|c|c|c|c|c|c|}
\hline ine & -0.18 & - : :2. & -1 & $B_{=11}$ & $10 x$ & - ar & $W_{s=1}$ & os $x$ & r.s. & -1.32 & . $4 x$ & Fer \\
\hline$z$ & Esmoses & $-1 \operatorname{ses} 6$ & & 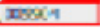 & 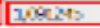 & thathin & $L=8 x-30$ & 511.06 & - Ecroust & atsorsu & acsects & $-\sin x$ \\
\hline ฐ & $-2,40305$ & 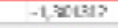 & & $\operatorname{axcin}$ & $-1 \operatorname{sen} x$ & 2., & 1,780 & 4, normes & 2nocmin & atais & assess: & $20 x=8$ \\
\hline 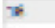 & - sivars & -1 crists & $\therefore$ & 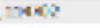 & $x \rightarrow+\infty=1$ & -4, & axtins & 5,11200 & andeis & aesem & 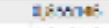 & $-250 x+6$ \\
\hline 2 & $-200 \mathrm{ne}$ & -1 these & s. & $3 \times n$ & 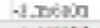 & $-2,>0 \times 36$ & $\operatorname{ang} x$ & segers & 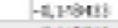 & atson & ansent: & owoset \\
\hline 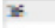 & -2,AJEC: & 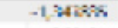 & \& 7 & 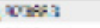 & $-1,4$ icse & $054: 12$ & 1 sख⿰ & L,0k515i & -4, 15ath & asizeno & 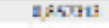 & crmis \\
\hline$\rightarrow$ & sroun: & -1 asins & s. & $\operatorname{sen}(4)$ & A.sunst & - -y,olls. & Ansers & sobres: & $-4100 x$ & asion & aहnes. & Cunes \\
\hline 54 & 1,:354785 & - LIFABA & & redia & 2,4293 & 3,4ล5a:0 & $-1.20 \mathrm{~s}$ क & E,OAOSA4 & 2. 12mes & QATent & \#:FASI & c notsil \\
\hline 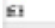 & $-4,6,004$ & $-1 \times \sin$ & $\leq 0$ & voses 4 & $-1,76 \times 6$ & 106259 & aperan: & sobsero & -4, 16:as! & asirne & $2 \pi \cos 1$ & cyritso \\
\hline$\pi$ & 2,:7:248: & 135485 & & 200055 & 1,됴:300 & 2.962054 & $3 \sin x$ & zoseser: & 2,19518: & adrus & 23asan & $\cos 354$ \\
\hline E1 & A,3ert: & Creasi & $2=$ & $x \in$ & C.AOSSI & - & -IYE!! & $200-104 i$ & 2,20158 & $\alpha+\sqrt{2} \approx$ & Das45: & C. $104: 56$ \\
\hline$\equiv$ & 1,rimes: & crases & & $2 x$ & Costoces & $4 x 073$ & fane:s & zodoes: & E, $15 \times$ & $0,4: 2004$ & 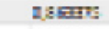 & C netra \\
\hline 표 & 2a179es: & c:senses & & nesia & 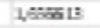 & 2.25013 & 4 nutr. & 2004 & armuc: & a.asese & 20541as & c messes \\
\hline xs & zasecu & c soseca & & 2005 & 2008:8 & 1.2x:5 & \&orus & noreas: & E,1006s & 0a:4ax & naseca & cocsse \\
\hline IIV & 2,090851 & Cxnas & 21 & Imeal & 2,73arts & Q earse & 43001 & 205ese: & 2,15xes & 0,30009 & 2.3man: & C mocess \\
\hline 로 & $-8,365006$ & tonitu & & astian & -1도어연 & Q320136 & -1 uns & socosas & $-2,1$ sosk & azsurus & nawe: & 0.000902 \\
\hline as: & 2,3005m & cxanocas & 23 & 28862 & C. 25000 & Lot:1 & 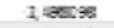 & 20000: & 2,1s9uc: & $027 x=5$ & 2, a mexec: & corcrose \\
\hline 29 & ther & toxits & & soxica & -1.46800 & 1.5025 & $-1, \operatorname{san} 45 d$ & solusit & -2,13ra: & $02 \times a=0$ & aikess: & 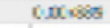 \\
\hline ss & ES:EKa & $\cos 0$ & & 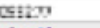 & 1, wescoso & $330 \mathrm{KL}$ & -13850 & zoeens. & $2,006 a$ & azsona & agkm & 6. 0083 \\
\hline$x^{2}$ & $-8,3654$ & -104661. & & tente & to.0000 & 1 astiso & $-1,000 \%$ & swets. & - swander & azisoset & aहsme & coneser \\
\hline 11 & -MHLE- & -1 xese & בx & octa & -1 lowand & 1, vers & Lesa & sobers & - wousals & azions & acusies & 10000044 \\
\hline Iso & trasoos & -1 susa & & xxels? & 1, LUEC: & Q ब्रम3: & $2364: 6$ & Lockes: & 2,00034 & 02.230 & 28:ase! & C. DESOEA \\
\hline xat & caris.s & $-1.2 \pi \%$ & & wite & toribuf & -1, seans & $x \times w$ & $200 \mathrm{e}=6$ & - sucostinar & assoces & acsest & Cuses \\
\hline 253 & -2.601 & $-1230 \mathrm{~m}$ & & 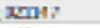 & -1.536000 & 1 xes & L4M & sovesit & -80605 & 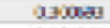 & mavers & cossies \\
\hline$x:$ & 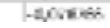 & 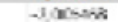 & & inots & 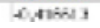 & $-2,34001$ & 20106 & SMOMM & Hatsero & axpen & abress & Cracers \\
\hline
\end{tabular}

Figure4. Example Table of Determination of vectors

Where $\overrightarrow{U_{s_{j} i}}=x_{s_{j} i} \overrightarrow{\boldsymbol{\imath}}+y_{s_{j} i} \overrightarrow{\boldsymbol{J}}+z_{s_{j} i} \overrightarrow{\boldsymbol{k}}$.

Where $x_{s_{j} i}, y_{s_{j} i}, z_{s_{j} i}$ are the value of position of sensor $j$ in the time on the $i$ axis $O X, O Y, O Z$ respectively.

To calculate the long and the angel of vectors we need to define a coordinates-center. For our paper we assume that the coordinates-center is the $(0,0,0)$. We can define the suitable coordinates-center corresponding to the study field. In this case the long of a vector is defined as

$$
\left|\overrightarrow{U_{s_{j} i}}\right|=\sqrt{\left(x_{s_{j} i}\right)^{2}+\left(y_{s_{j} i}\right)^{2}+\left(z_{s_{j} i}\right)^{2}}
$$

And the angle between two successively vectors is as following

$$
\theta_{s_{j} i}=\arccos \left(\frac{\overrightarrow{U_{s_{j} i}} \cdot \overrightarrow{U_{s_{j} i+1}}}{\left|\overrightarrow{U_{s_{j} i}}\right| \cdot\left|\overrightarrow{U_{s_{j} i+1}}\right|}\right)
$$

We restore the resulting values in new table like in Figure (Figure 5) 


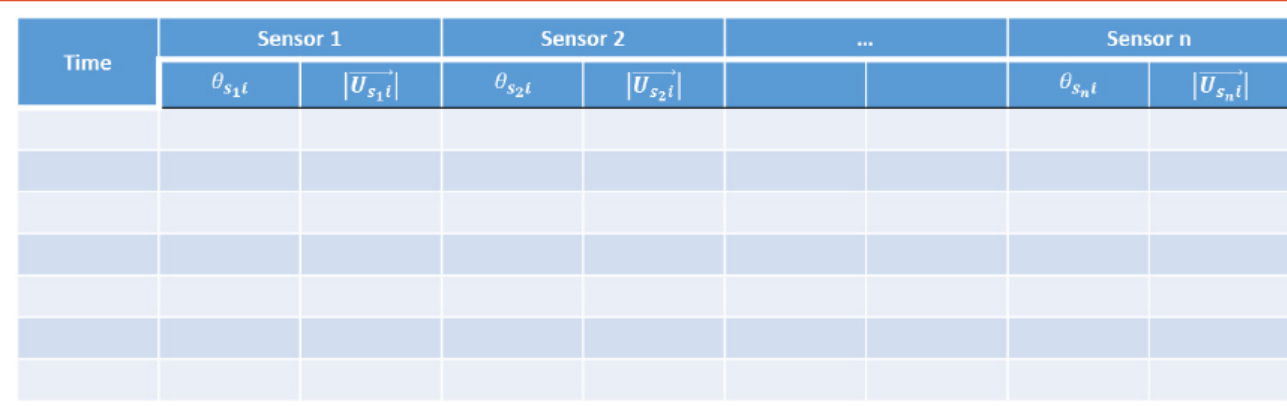

Figure5. Derived Table

Now to determinate the similarities between sensors we start to define a regression to execute the analysis acoording this regression.

We have design the vector model with aim to be able to define a linear regression, which maybe be able to make the analysis more simply. Figure 6 shows the representation of motion for sensors according two directions. The first one is according to each sensor in all measuring times and the other one according to each time for all sensors.
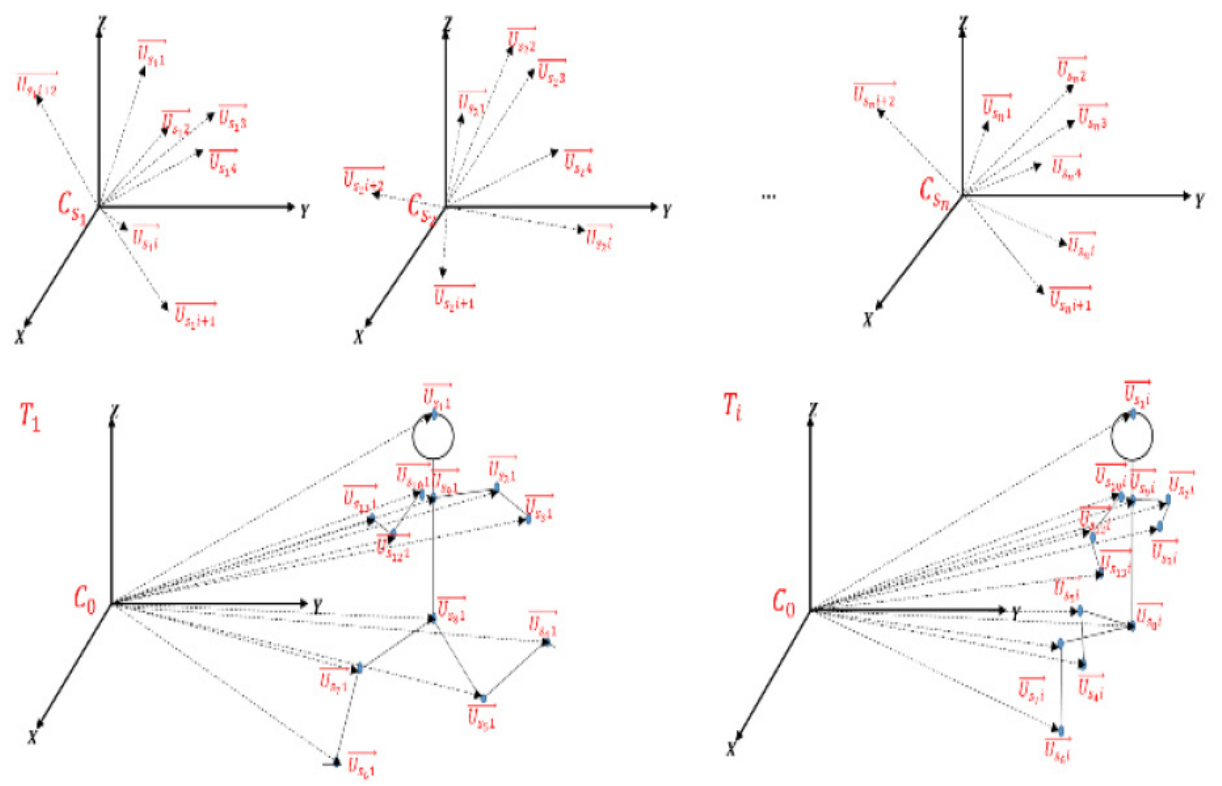

Figure6. Motion Vectors

\section{Definition of Linear Regression}

The two attributes $\left(\boldsymbol{\theta}_{\boldsymbol{s}_{j} \boldsymbol{i}}\left|\overrightarrow{\boldsymbol{U}_{\boldsymbol{s}_{\boldsymbol{j}}}}\right|\right)$ of each sensors in resulted table are the two variable of our linear regression $\boldsymbol{R}_{\boldsymbol{S}_{\boldsymbol{j}}} \cdot \boldsymbol{\theta}_{\boldsymbol{s}_{\boldsymbol{j}} \boldsymbol{i}}$ is the dependent variable. The equation of our regression is defined as following:

$$
\operatorname{Re}_{s_{j}}=\theta_{s_{j} \bar{i}}=a_{s_{j}}+b_{s_{j}}\left|\overrightarrow{U_{s_{j} \bar{i}}}\right|
$$

Where

$$
\begin{gathered}
b_{S_{j}}=\frac{n \sum_{i}\left|\overrightarrow{U_{s_{j}}}\right| \theta_{s_{j} i}-\left(\sum_{i}\left|\overrightarrow{U_{s_{j}}}\right|\right)\left(\sum_{i} \theta_{s_{j} i}\right)}{\left.n \sum_{i}\left|\overrightarrow{U_{s_{j}}}\right|\right|^{2}-\left(\sum_{i}\left|\overrightarrow{U_{s_{j}}}\right|\right)^{2}} \\
a_{S_{j}}=\frac{\sum_{i} \theta_{s_{j} i}-b_{s_{j}} \sum_{i}\left|\overrightarrow{U_{s_{j} i}}\right|}{n}
\end{gathered}
$$


According this regression we achieve the following result of our example (Figure 7).

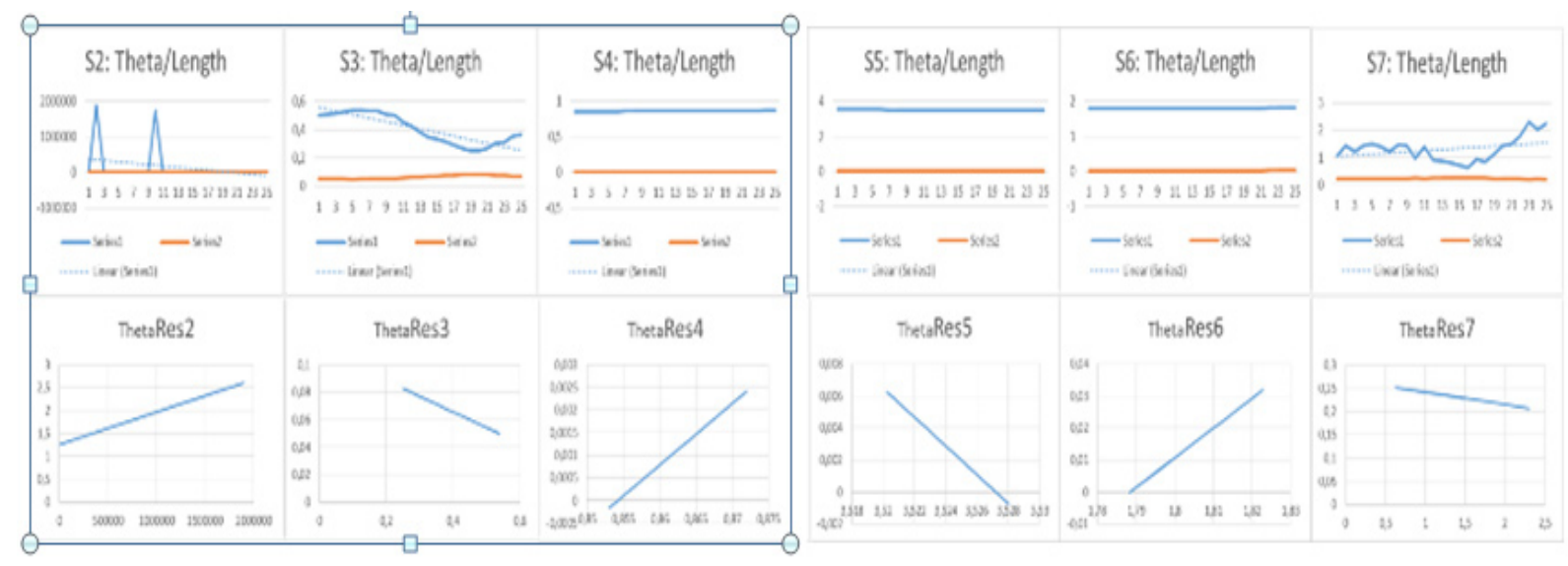

Fig7. Illustration of regressions / one Angle

From this anaylsis we find that there is a big similarity between sensor 4 and 6 . We find too that the similarity between 5 and 4 or 6 is big too but in inverse direction.

For more determination of the similarity between sensors we define more regressions according other angels $(\alpha, \beta, \gamma)$.

\section{More Linear Regressions}

To clarify the similarities more between the sensors we define more linear regressions according the angles bwteen vectors and coordinates-axes $(\alpha, \beta, \gamma)$ (See Figure 8).

Where

$\alpha_{s_{j} i}$ : Angle between a vector $\overrightarrow{U_{s_{j}} i}$ and X-Axis

$\beta_{s_{j} i}$ : Angle between a vector $\overrightarrow{U_{s_{j} i}}$ and Y-Axis

$\gamma_{s_{j} i}$ : Angle between a vector $\overrightarrow{U_{s_{j} i}}$ and Z-Axis

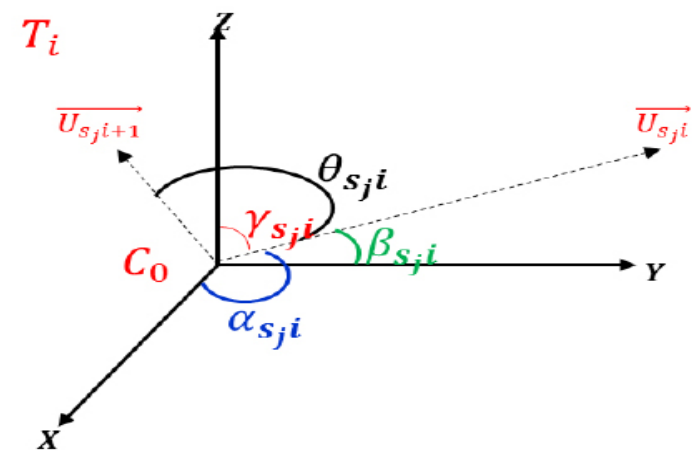

Fig8. Illustration of Angles

We define now 3 other linear regressions

$$
\begin{aligned}
R e_{\alpha_{j}}: \alpha_{s_{j} i} & =a_{\alpha_{j}}+b_{\alpha_{j}}\left|\overrightarrow{U_{s_{j}} i}\right| \\
R e_{\beta_{j}}: \beta_{s_{j} i} & =a_{\beta_{j}}+b_{\beta_{j}}\left|\overrightarrow{U_{s_{j}} i}\right| \\
R e_{\gamma_{j}}: \gamma_{s_{j} i} & =a_{\gamma_{j}}+b_{\gamma_{j}}\left|\overrightarrow{U_{s_{j} i}}\right|
\end{aligned}
$$

Volume 1 
Where

$$
\begin{gathered}
b_{\alpha_{j}}\left(b_{\beta_{j}} \backslash b_{\gamma_{j}}\right)=\frac{n \sum_{i}\left|\overrightarrow{\boldsymbol{U}_{s_{j} i}}\right| \alpha_{\boldsymbol{s}_{j} i}\left(\beta_{\boldsymbol{s}_{j} i} \backslash \gamma_{s_{j} i}\right)-\left(\sum_{i}\left|\overrightarrow{\boldsymbol{U}_{s_{j} i}}\right|\right)\left(\sum_{i} \alpha_{\boldsymbol{s}_{j} i}\left(\beta_{\boldsymbol{s}_{j} i} \backslash \gamma_{s_{j} i}\right)\right)}{n \sum_{i}\left|\overrightarrow{\boldsymbol{U}_{s_{j} i}}\right|^{2}-\left(\sum_{i}\left|\overrightarrow{\boldsymbol{U}_{s_{j} i}}\right|\right)^{2}} \\
a_{\alpha_{j}}\left(a_{\beta_{j}} \backslash a_{\gamma_{j}}\right)=\frac{\sum_{i} \alpha_{s_{j} i}\left(\beta_{s_{j} i} \backslash \gamma_{s_{j} i}\right)-b_{\alpha_{j}}\left(b_{\beta_{j}} \backslash b_{\gamma_{j}}\right) \sum_{i}\left|\overrightarrow{\boldsymbol{U}_{s_{j} i}}\right|}{n}
\end{gathered}
$$

According these regression we find the following illustration of similarities of our example (Figure 9).

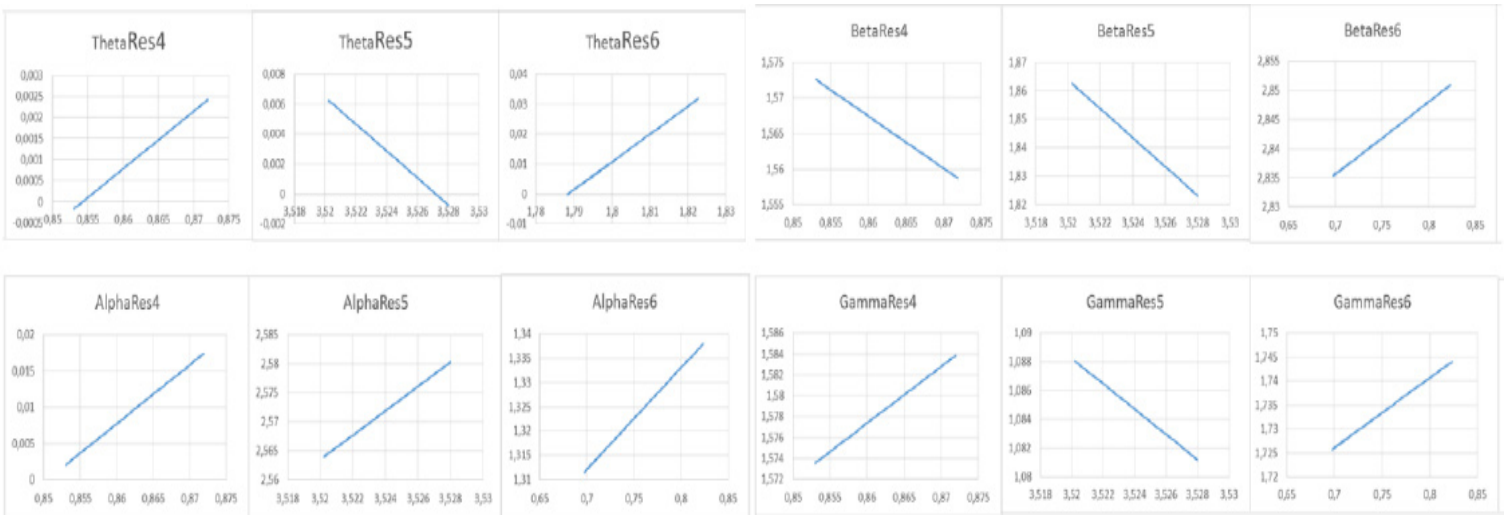

Fig9. Illustration of regressions / many angles

Now we come to phase, where we can compute the coefficient of sensors according quadratic deviation and quadratic residuum on the 4 angles (4 regressions) and the long of the vectors.

After execuation of this analysis we get the following results of our example:

\section{- Coefficient of sesnors 4 and 5}

- About $98 \%$ of the variation of Lengths of the sensor 4 can be explained with sensor 5

- About $99 \%$ of the variation of Thetas of the sensor 4 can be explained with sensor 5

- About $97 \%$ of the variation of Alphas of the sensor 4 can be explained with sensor 5

- About $96 \%$ of the variation of Betas of the sensor 4 can be explained with sensor 5

- About $96 \%$ of the variation of Gammas of the sensor 4 can be explained with sensor 5

\section{- Coefficient of Sensor 4 and Sensor 6}

- About 74\% of the variation of Lengths of the sensor 4 can be explained with sensor 6

- About $80 \%$ of the variation of Thetas of the sensor 4 can be explained with sensor 6

- About $16 \%$ of the variation of Alpha of the sensor 4 can be explained with sensor 6

- About $13 \%$ of the variation of Beta of the sensor 4 can be explained with sensor 6

- About 38\% of the variation of Gamma of the sensor 4 can be explained with sensor 6

\section{- Coefficient of Sensor 5 and Sensor 6}

- About $80 \%$ of the variation of Lengths of the sensor 5 can be explained with sensor 6

- About 79\% of the variation of Thetas of the sensor 5 can be explained with sensor 6 
- About $17 \%$ of the variation of Alpha of the sensor 5 can be explained with sensor 6

- About $10 \%$ of the variation of Beta of the sensor 5 can be explained with sensor 6

- About $25 \%$ of the variation of Gamma of the sensor 5 can be explained with sensor 6

From this result we can find that only according to one angle (regression) have we to say that the similarity between sensor 4, 5 and 6 is very big (good) but according to the other angles (regressions) we can say that the similarity is only very big between sensor 4 and 5 .

\section{CONCLUSION}

The recognition of motion needs complex equations to determine the the type of motion. It is impossible to define a linear regression direct of the measured data (Position, Velocity, Acceleration, Orintation, etc.), which illustrates the motion data in relevant form. We have define our new model according angles and long of defined vector, to be able to define a linear re-regression, which will be used to analyze the derived measured data and to extract the similarities between different measure resources (sensors).

Only with using data analysis (Big Data Analysis -in care systems with sensors -) we wanted to get results that could give us decision about which sensors can be eliminated. We could extract the similarities between the sensors according to their measured data, which illustrated through the defined model with its linear regressions. According to these similarities and other factors like placement of sensors we can determine the sensors, which can be eliminated.

The motion in walk case can be recognized by two angles, thrust and approach angles. From this idea we defined a vector connected with the sensors to be able to calculate the angles between this vectorand coordinates axes and too successively vector. We found that we need to define other factor to recognize the other motion types like sit, stand, etc. This factor is the long of the vector.With this idea we can illustrate the principle and the results of the distance matrix analysis.

The methodology (ReSeVA) that presented in this article can be applied not only according to measurements of the same type like position-, acceleration- ... or velocity-measurements but too according to mix of measurements of different types. That means, we can define a vector, which contains values of different measurement types (position-, acceleration- ... and velocity-measurements all together in on vector).

This idea allows us to apply our regression analysis on $n$ dimensional vectors ( $n D$-vectors). This analysis has following properties:

1- It has uncomplex computations

2- It is applicable on all data models

3- It is applicable on raw data

4- It is applicable on derived data

5- It is applicable on $2 D, 3 D, \ldots, n D$ spaces

6- It is implementable by using data analysis tools, like tools in all database systems, R, Matlab, Excel, etc.

7- It is implementable and intgrable in all recogniation systems

After the determination of unneeded sensors we have to test the result of the applied recogniation system without the values of these determined sensors. Basend on this verification we can extract more information that can be improve the result of our analysis. 


\section{ACKNOWLEDGMENT}

We would like to thank our colleague in Database and information systems department at university of Rostock for their notes. Moreover, we would like to express our gratitude IIE-SRF/USA organization for its support scientists at risk and its scholarships.

\section{REFERENCES}

1. Englert, F.; Diaconita, I.; Reinhardt, A.; Alhamoud, A; Meister, R.; Backert, L.; Steinmetz, R.: Reduce the Number of Sensors: Sensing Acoustic Emissions to Estimate Appliance Energy Usage. In 5th ACM Workshop on Embedded System for Energy -Efficient Building. 2013.

2. Xiang, Y.; Tang, Y.; Zhu, W.: Mobile sensor network noise reduction and re-calibration using Bayesian network. In Atmospheric Measurement Techniques Discussions, pp. 8971-9008, Copernicus Publications on behalf of the European Geosciences Union, 2015.

3. Kim, J.; Seol, Y.; Lee, Jehee: Realtime Performance Animation Using Sparse 3D Motion Sensors. In Proceedings of Motion in Games (MIG), Rennes, France, 2012.

4. Badler, N. I.; Hollick, M. J.; Granieri, J. P.: Real-Time Control of a Virtual Human Using Minimal Sensors. In Presence, Volume 2, Issue 1, 1993, pp. 82-86. URL: http://www.mitpressjournals.org/loi/pres.

5. Altun, K.; Barshan, B.: Human Activity Recognition Using Inertial/Magnetic Sensor Units. In c SpringerVerlag Berlin Heidelberg, HBU 2010, LNCS 6219, 2010, pp. 38-51.

6. Jones, S.; Shao, L.: Linear Regression Motion Analysis for Unsupervised Temporal Segmentation of Human Actions. In Department of Electronic and Electrical Engineering,the University of Sheffield, Mappin St, Sheffield, S1 3JD, UK.

7. Chai, J; Hodgines, J.K.: constraint-based motion optimization using a statistical synamic model. In ACM Transactions on Graphics, SIGGRAPH, 2007.

8. Chai, J; Hodgines, J.K.: Performance animation from low-dimensional control aignals. In ACML Transactions on Graphics, SIGGRAPH, 2005.

9. Feng, W.W.; Kim, B.U., Yu, Y.: Real-time data driven deformation using kernel canonical correlation analysis. In ACM Transactions on Graphics, SIGGRAPH, val 27 (3), 2008

10. Huang, H.; Yin, K.; Zhao, L.; Qi, Y.; Yu, Y.; Tong, X.: Detail-preserving controllable deformation from spares examples. In IEEE Transactions on Visulaization and Computer Graphics, pp. 1215 - 1227, 2012.

11. Liu, G.; Zhang, J.; Wang, W., McMillan, L.: Human motion estimation from a reduced marker set. In ACM Syposium on Interactive 3D graphics and games, pp. 35-42. 2006.

12. Liu, H.; Wei, X.; Chai, J.; Ha, I.; Rhee, T.: Realtime human motion control with a small number of intertial sensors. In Symposium on Interactive 3D Graphics and Games, pp. 133 - 140. 2011.

13. Bazinga, T.; Chen, C.; Li, G.; Ngo, P.; SunLiu, C.: Motion Sensing Technolog. In Management of Technology - E 103, report June 2. 2011.

14. Premaratne, P.: Human Computer Interaction Using Hand Gestures. In Cognitive Science and Technology, DOI 10.1007/978-981-4585-69-9_2, Springer Science+Business Media Singapore, 2014.

15. Jo, H.; Yu, H.; Kim, K.; Hwan Sung, Y.: Motion Tracking System for Multi-User with Multiple Kinects. In International Journal of u- and e- Service, Science and Technology, Vol.8, No. 7, pp.99-108, 2015 
16. Xie, L.; Kumar, M.; Cao, Y.; Gracanin, D.; Quek, F.: Data-driven Motion Estimation with Low-Cost Sensors. In Computer Science Department Virginia Polytechnic Institute and State University, United States.

17. Scholz, D.; Friedmann, M.; Stryk, O: Fast, Robust and Versatile Humanoid Robot Locomotion with Minimal Sensor Input. In Simulation, Systems Optimization and Robotics Group Technische Universitat Darmstadt.

Citation: Ammar Suhail Balouch, "Reducing Sensors according to a VectorsAnalysis of stored measurements (ReSeVA)". American Research Journal of Computer Science and Information Technology, Volume 1; pp:1-10

Copyright (c) 2016 Ammar Suhail Balouch, This is an open access article distributed under the Creative Commons Attribution License, which permits unrestricted use, distribution, and reproduction in any medium, provided the original work is properly cited. 